\title{
MiR-I52 functioning as a tumor suppressor that interacts with DNMTI in nasopharyngeal carcinoma
}

This article was published in the following Dove Press journal:

OncoTargets and Therapy

\author{
Zhi-Wei Lu ${ }^{1,2, *}$ \\ Ming-Yu $\mathrm{Du}^{2, *}$ \\ Lu-Xi Qian ${ }^{1,2}$ \\ Nan Zhang ${ }^{2}$ \\ Jia-Jia $\mathrm{Gu}^{2}$ \\ Kai Ding ${ }^{3}$ \\ Jing $\mathrm{Wu}^{2}$ \\ Hong-Ming Zhu ${ }^{2}$ \\ Xia $\mathrm{He}^{1,2}$ \\ Li Yin ${ }^{1,2}$
}

'The Fourth Clinical School of Nanjing Medical University, Nanjing, Jiangsu, China; ${ }^{2}$ Department of Radiation Oncology, Jiangsu Cancer Hospital, Jiangsu Institute of Cancer Research, Nanjing Medical University Affiliated Cancer Hospital, Nanjing, Jiangsu, China; ${ }^{3}$ Department of Radiation Oncology, Suqian First Hospital, Suqian, Jiangsu, China

*These authors contributed equally to this work
Correspondence: Xia He; Li Yin The Fourth Clinical School of Nanjing Medical University, I 40 Hanzhong Road, Nanjing 210000, Jiangsu, China

Tel +86 I360I4585 I 8; +86 I39I3909043 Email hexiabm@I63.com; yinli_2012@126.com
Background: In recent years, miR-152 has been dysregulated in a variety of tumors and used as a tumor suppressor. Nevertheless, its role in nasopharyngeal carcinoma (NPC) remains unidentified.

Materials and methods: Real-time quantitative PCR (polymerase chain reaction) was performed to analyze the expression of miR-152 in NPC cell lines. MiR-152 expression profiles in NPC tissues were obtained from Gene Expression Omnibus (GEO GSE36682). The effect of miR-152 on the invasion and proliferation of NPC cells was determined through cell invasion, wound healing, and cell viability assays. Apoptosis was examined by flow cytometry, and Western blot was performed to measure expression of the target gene. Pyrosequencing was used to detect the methylation level of NPC cells.

Results: In this study, miR-152 was downregulated in the NPC tissues and cell lines. When miR-152 was enhanced, the invasion and migration of NPC cells were inhibited. However, miR-152 had no effect on the proliferation of NPC cells. Luciferase reporter gene analysis was performed, and the results showed that DNMT1 (DNA (cytosine-5)-methyltransferase 1) is a direct target of miR-152 in NPC cells. DNMT1 downregulation and miR-152 overexpression both reversed the effects of miR-152 inhibition on the NPC cells. In addition, miR-152 expression increased as a result of the inhibition of the methylation level of miR-152 when DNMT1 expression was downregulated.

Conclusion: The overexpression of miR-152 inhibited the migration and invasion of NPC cells by targeting DNMT1. Furthermore, DNMT1 regulated miR-152 expression through DNA methylation. Overall, the novel miR-152-DNMT1 regulatory circuit may provide better understanding of the pathogenesis of NPC and new epigenetic therapeutic target in NPC.

Keywords: miR-152, DNMT1, NPC, methylation

\section{Introduction}

Nasopharyngeal carcinoma (NPC) is a prevalent head and neck cancer in Southeast Asia, and is closely related to Epstein-Barr virus infection. ${ }^{1}$ Although NPC is sensitive to chemoradiation, the 5-year survival rates of individuals with NPC remain unsatisfactory. Local recurrence and distant metastasis are the main cause of failure. ${ }^{2,3}$ Therefore, the molecular mechanism of NPC pathogenesis must be explored.

MicroRNAs (miRNAs) are a group of highly conserved, noncoding, and single stranded small RNA (ribonucleic acid) molecules with lengths of 18-25 nucleotides. MiRNAs inhibit gene expression in combination with the $3^{\prime}$-UTR sequences of targeted mRNAs (messenger RNAs). ${ }^{4}$ Previous studies suggested that some miRNAs are involved in cancer formation, as they control cell functions, such as proliferation, 
differentiation, and apoptosis. ${ }^{5}$ So far, miR-152 is dysregulated in various tumors and tumor suppressors, ${ }^{6}$ such as gastric cancer, ${ }^{7}$ colorectal cancer ${ }^{8}$ hepatocellular carcinoma, ${ }^{9}$ and prostate cancer. ${ }^{10}$ In addition, miR-152 is more downregulated in NPC than in normal tissue, indicating that it has potential as a tumor suppressor. ${ }^{11}$ Nevertheless, the role of miR-152 in NPC remains unknown.

DNMT1 (DNA (cytosine-5)-methyltransferase 1) is a type of methyltransferase responsible for maintaining DNA methylation during DNA replication. ${ }^{12}$ Several studies showed that DNMT1 expression considerably increases in NPC, ${ }^{13}$ and DNMT1 overexpression leads to an increase in DNA methylation; this increase is often associated with a poor prognosis of NPC. ${ }^{14}$ Furthermore, the upregulation of DNMT1 expression may promote DNA methylation, thereby reducing miR-152 expression. ${ }^{15}$ However, the relationship between miR-152 and DNMT1 in NPC remains unclear.

The purpose of this study is to investigate the role of miR-152 in the regulation, proliferation, invasion, and migration of NPC, and the mechanism of its interaction with DNMT1.

\section{Materials and methods}

\section{Patients and samples}

The MiR-152 expression profiles in NPC tissues were obtained from Gene Expression Omnibus (GEO GSE36682; www.ncbi.nlm.nih.gov/geo/). In addition, GEO2R was used for the comparison between the miR-152 expression levels of tumors and normal tissues.

\section{Cell culture}

Six NPC cells (CNE1, CNE2, 5-8F, 6-10B, HNE1, and SUNE1) and NP69 (ATCC, Manassas, VA, USA) were provided by the Research Center of Clinical Oncology of the Affiliated Jiangsu Cancer Hospital, Nanjing Medical University, in Nanjing, China. The cell lines were cultured in RPMI 1640 (Corning, Manassas, VA, USA) supplemented with 5\% fetal bovine serum (FBS; Gibco, Waltham, MA, USA) at $37^{\circ} \mathrm{C}$ in a humidified chamber supplemented with $5 \% \mathrm{CO}_{2}$.

\section{RNA extraction and real-time qPCR}

Total RNA was isolated with TRIzol reagent (Invitrogen, Carlsbad, CA) according to manufacturer's instructions. Then, $2 \mu \mathrm{g}$ of total RNA was reverse transcribed into cDNA with a reverse transcription reagent kit (Promega, Madison, WI, USA). The expression levels of mature miRNAs were amplified with SYBR Green quantitative real-time polymerase chain reaction (qRT-PCR) on an ABI7300 real-time PCR machine (Applied Biosystems, Waltham, MA, USA). The expression of miR-152 was normalized to the expression of U6. The primers for human miR-152 and U6 were synthesized by Ribobio (Guangzhou, China). The expression of DNMT1 was normalized to the expression of $\beta$-actin. The expression levels of DNMT1 and $\beta$-actin were examined, and the following specific primers were used: 5'-AGGCGGCTCAAAGATTTGGAA-3' and 5'-GCAGAAATTCGTGCAAGAGATTC-3' for DNMT1, and 5'-GGACTTCGAGCAAGAGATGG-3' and 5'-AGCACTGTGTTGGCGTACAG-3' for $\beta$-actin. All reactions were performed in triplicate for each sample. Fold changes for miR-152-3p and DNMT1 expression levels were calculated through the $2-\Delta \Delta \mathrm{Ct}$ method.

\section{Cell transfection}

MiR-152-3p mimic/inhibitor and miR negative control (NC) were synthesized by RiboBio. DNMT1-siRNA and NC-siRNA were obtained from RiboBio. CNE-2 cells were transfected with miRNA mimic/inhibitor/DNMT1-siRNA with Lipofectamine 2000 (Invitrogen) for $48 \mathrm{~h}$. To investigate the relationship between miR-152 and DNMT1, MiR-152 inhibitor/NC inhibitor and DNMT1-siRNA/NC-siRNA were cotransfected into $\mathrm{CNE}-2$ cells at the same time. To determine the transfection efficiency of miR-152 mimic/inhibitor/DNMT1siRNA, the expression of miR-152/DNMT1 was assessed with a qRT-PCR detection system and Western blot apparatus.

\section{Cell viability assay}

Cell proliferation was assessed by cell counting kit-8 (CCK-8; Dojindo, Kumamoto Prefecture, Kyushu, Japan). After $48 \mathrm{~h}$ of transfection, cells were collected and reseeded in 96 -well plates $\left(3 \times 10^{3}\right.$ cells per well) for cell viability assay. The reaction solution $(10 \mu \mathrm{L})$ was added into cultured cells in $100 \mu \mathrm{L}$ of culture medium and incubated at $37^{\circ} \mathrm{C}$ for $40 \mathrm{~min}$. Cell proliferation was detected at 24,48 , and $72 \mathrm{~h}$ with CCK-8 (Dojindo). Optical density was determined at a wavelength of $450 \mathrm{~nm}$.

\section{Cell wound healing assay}

We collected CNE-2 cells in logarithmic phase, and seeded them into six-well plates $\left(1.5 \times 10^{5}\right.$ cells per well). When cells reached $80 \%-90 \%$ confluence, similar-sized wounds were scratched on the surface of the well with $200 \mu \mathrm{L}$ sterile micropipette tips. Cells were then washed three times with phosphate-buffered saline (PBS) and incubated in Dulbecco's Modified Eagle's Medium with 1\% fetal bovine serum (FBS). Photographs were taken at indicated times ( 0 and $24 \mathrm{~h})$ using an inverted microscope (Olympus, Tokyo, Japan).

\section{Cell invasion assay}

Invasion assays were performed with Transwell chambers, each with a pore size of $8 \mu \mathrm{m}$ (BD Biosciences, San Jose, CA, USA). 
Transfected CNE-2 cells were cultured for $48 \mathrm{~h}$. The cells in $200 \mu \mathrm{L}$ of serum-free media were added to the upper compartment $\left(2 \times 10^{4}\right.$ cells per well) of the chamber (Corning), and the lower compartment was filled with $500 \mu \mathrm{L}$ of RPMI-1640 and 20\% FBS. After $36 \mathrm{~h}$ of incubation, the cells on the upper surface were removed, whereas the cells that migrated to the bottom surface were fixed in $4 \%$ methanol and stained with crystal violet. Six random fields of cells were counted in each well under a microscope at a magnification of $100 \times$.

\section{Flow cytometric analysis}

After $48 \mathrm{~h}$ of transfection, CNE-2 cells were trypsinized and collected at $48 \mathrm{~h}$. Cell pellets were rinsed once with PBS and fixed overnight in $70 \%$ ice-cold ethanol at $-20^{\circ} \mathrm{C}$. The cells were then resuspended in $\mathrm{PBS}$, stained with propidium iodide containing RNase A for $30 \mathrm{~min}$ at $37^{\circ} \mathrm{C}$, and analyzed by flow cytometry. Each assay was repeated at least three times.

\section{Western blot analysis}

After $48 \mathrm{~h}$ of transfection, cells were extracted and prepared in modified radioimmunoprecipitation assay buffer (Beyotime, Shanghai, China). Total protein was extracted, and protein concentration was quantified with a bicinchoninic acid (BCA) protein assay kit (Beyotime). A total of $20 \mathrm{mg}$ of protein from each sample was used for Western blot analysis. The primary antibodies used in this study included monoclonal anti-DNMT1 (1:1,000; Cell Signaling Technology, Danvers, MA, USA), and $\beta$-actin was used as loading control. Immunoreactive bands were visualized with ECL (enhanced chemiluminescence) detection reagent (Millipore, Billerica, MA, USA). All data analyses were repeated three times independently.

\section{Luciferase reporter construction and luciferase assays}

Human DNMT1 3'UTR regions containing predicted binding site for miR-152-3p were constructed on pmirGLO vectors, and a sequence of pri-miR-152-3p was inserted into pCDNA3.1 plasmid. The CNE-2 cells were inoculated into 24-well plates and cultured for $24 \mathrm{~h}$. The cells were subsequently co-transfected with $\mathrm{Wt} / \mathrm{Mut}$ reporter plasmid $(0.2 \mu \mathrm{g})$ and miR-152 mimic/miR-NC. Cells were harvested $48 \mathrm{~h}$ after transfection. Luciferase activities were analyzed using the dual-luciferase reporter assay system according to manufacturer's protocol.

\section{Experiment with 5-aza-2'-deoxcycytidine}

DNA-hypomethylating drug 5-aza-2'-deoxycytidine (SigmaAldrich, St Louis, MO, USA) was used to verify the regulation of DNA methylation in the NPC cells. The cells were treated with 5 -aza-cdr $(0,10$, and $20 \mu \mathrm{mol} / \mathrm{L})$ for 3 days and with TSA $(100 \mathrm{ng} / \mathrm{mL})$ for an additional $24 \mathrm{~h}$. Cells were harvested for DNA and RNA extraction.

\section{DNA isolation and bisulfite conversion}

Genomic DNA was isolated from CNE-2 cells using a QIAamp DNA Mini Kit (Qiagen, Venlo, the Netherlands) according to the manufacturer's instructions. A Qiagen's EpiTect bisulfite kit was used for the bisulfite conversion of the DNA sample, and $1-2 \mu \mathrm{g}$ of DNA was diluted to $40 \mu \mathrm{L}$ with deuterium depleted water in $0.5 \mathrm{~mL}$ PCR tubes. Freshly prepared bisulfite conversion $(85 \mu \mathrm{L})$ and DNA protection solution (PB $15 \mu \mathrm{L})$ was then added to the solution. After mixing, the reaction tube was placed in PCR instrument at $95^{\circ} \mathrm{C}$ for $5 \mathrm{~min}$ and at $60^{\circ} \mathrm{C}$ for $1 \mathrm{~h}$ for five cycles. The bisulfite-converted DNA was stored at $20^{\circ} \mathrm{C}$.

\section{Pyrosequencing}

The miR-152 primers for the pyrosequencing assays were designed in PyroMark ${ }^{\circledR}$ Assay Design SW 2.0. The design contained a sequencing primer and an amplification primer set in which one primer was biotinylated. Bisulfite-converted DNA was amplified with PyroMark ${ }^{\circledR}$ PCR kit on a thermal cycler as follows: $3 \mathrm{~min}$ at $95^{\circ} \mathrm{C}, 50$ cycles of $15 \mathrm{~s}$ at $95^{\circ} \mathrm{C}$, $20 \mathrm{~s}$ at $54^{\circ} \mathrm{C}$, and $30 \mathrm{~s}$ at $72^{\circ} \mathrm{C}$, then a $5 \mathrm{~min}$ final extension stage at $72^{\circ} \mathrm{C}$. Subsequently, we isolated single-strand DNAs and performed pyrosequencing assays on PyroMark ${ }^{\circledR}$ Q96 according to the manufacturer's instructions. The degree of methylation of the target gene locus was analyzed with PyroMark $^{\circledR}$ Q96 Software (Sequencing section containing eight CpG sites: GGAGGCCACGCGCGAGAGGGGGCC GCGGGGCGGCGCGGAGG).

\section{Statistical analysis}

Student's $t$-test and one-way ANOVA were performed in GraphPad Prism 5.0 software and SPSS 13.0. Data are expressed as mean and standard deviation from at least three separate experiments. Differences were considered significant at a $P$-value of $<0.05$.

\section{Results \\ MiR-I52 is downregulated in NPC tissues and cell lines}

To confirm the role of miR-152 in tumorigenesis of NPC, we searched the GEO to obtain the expression profiles of miR-152 in NPC and normal tissues. As expected, the expression of miR-152 in NPC $(n=62)$ tissues was significantly lower than in normal tissue $(n=6)$, suggesting that the tumor suppressed miR-152 expression in NPC (GSE36682; Figure 1A). 
A

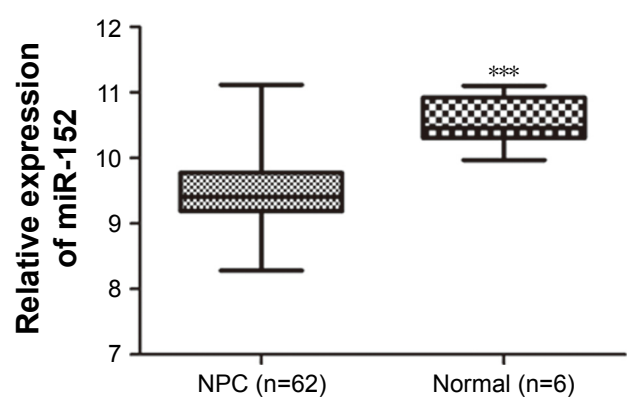

B

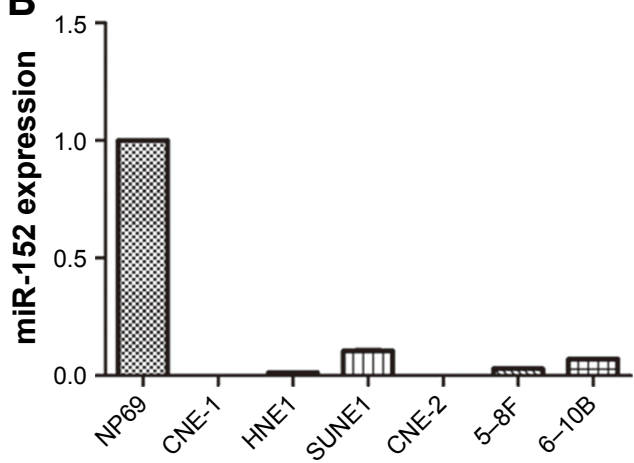

Figure I MiR-I52 was poorly expressed in the NPC tissues and cells. (A) Expression levels of miR-I52 in normal and NPC tissues. (B) Expression of miR-I52 was downregulated in NPC cells. $* * * P<0.01$.

Abbreviation: NPC, nasopharyngeal carcinoma.

For the verification of the hypothesis, the mRNA levels of miR-152 in the six NPC cell lines (5-8F, 6-10B, HNE1, SUNE1, CNE-1, and CNE-2) and NP69 were determined by qRT-PCR (Figure 1B). MiR-152 expression decreased in the six NPC cells, similar to that in NPC tissues. In addition, CNE-2 had the lowest miR-152 expression in the NPC cell lines. Hence, we used CNE-2 cells as a model for subsequent experiments.

\section{Overexpression of miR-I52 inhibits invasion and migration of NPC cells}

To clarify the role of miR-152 in NPC cells, we transfected CNE-2 cells with miR-152 mimics. By qRT-PCR, miR-152 expression considerably increased (Figure 2A). In the matrigel invasion assay, the number of cells invaded significantly decreased in the miR-152 mimic cells (Figure 2B). Furthermore, miR-152 had similar effects in the wound healing assays (Figure 2C). These results suggested that miR-152 inhibits the migration and invasion of NPC cells. However, cell proliferation was unaffected by the enhancement of miR-152 expression (Figure 2D). Similarly, no significant difference was observed between the mimic and control groups in the apoptosis assays (Figure 2E). In addition, when the expression of miR-152 was downregulated (Figure 2F), the invasion (Figure 2G) and migration (Figure $2 \mathrm{H}$ ) of NPC cells was promoted.

\section{Identification of DNMTI as a direct target of miR-I52 in NPC cells}

Identifying a target gene is crucial to the exploration of the mechanism of miR-152 in NPC cells. DNMT1 was selected as a potential target gene for miR-152. TargetScan software was used for the screening. To verify the relationship between miR-152 and DNMT1, we transfected CNE-2 cells with miR-152 mimic or negative control. By increasing expression of miR-152, we observed the downregulation of DNMT1 expression through qRT-PCR (Figure 3A) and Western blot analysis (Figure 3B). In addition, the 3'UTR of DNMT1 contained a conserved binding site for miR-152 (Figure 3C). To confirm that DNMT1 is a target gene of miR-152-3p in NPC cells, we constructed three vectors (pmirGLO, DNMT13UTR-WT, and DNMT1-3UTR-MUT) for the implementation of subsequent luciferase assay. Co-transfection of miR-152 and DNMT1-3'-UTR-WT significantly reduced the activity of luciferase, although this effect was not achieved in the mutant group (Figure 3D). Based on the above results, DNMT1 is an miR-152 target gene.

\section{DNMTI is involved in invasion and migration of NPC cells}

Although DNMT1 is a target for miR-152, and DNMT1 overexpression (31 of $32,97 \%$ ) can be observed in NPC tissues, ${ }^{13}$ the role of DNMT1 in NPC cells remains unclear. We transfected CNE-2 cells with DNMT1-siRNA or NCsiRNA, and performed qRT-PCR (Figure 4A) and Western blot experiments (Figure 4B) to verify whether DNMT1 expression was significantly reduced. After confirming the downregulation of DNMT1 expression, a matrigel invasion test and wound healing assays were conducted to validate the effect of DNMT1 on the invasion and migration ability of NPC cells. The results showed that the downregulation of DNMT1 expression significantly inhibited the migration (Figure 4C) and invasion (Figure 4D) of NPC cells.

Downregulation of DNMTI reversing the effect of inhibition of miR- 152 expression on invasion and migration of NPC cells

To further clarify that miR-152 regulates invasion of NPC cells, DNMT1, we co-transfected miR-152 inhibitor and DNMT1-siRNA into the CNE-2 cells. Then, miR-152 
A

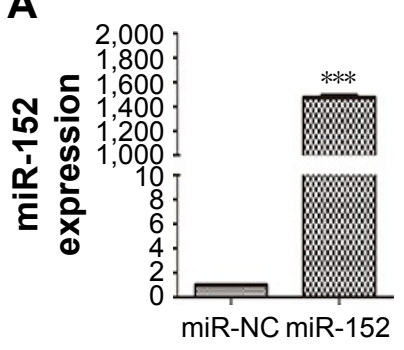

B

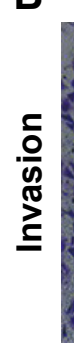

miR-NC

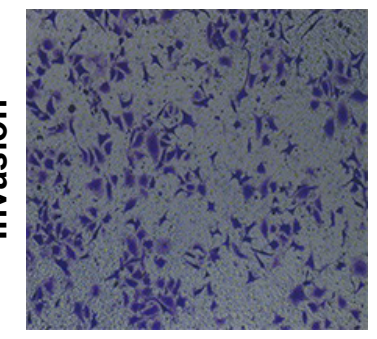

miR-152

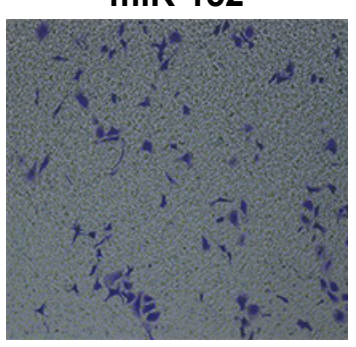

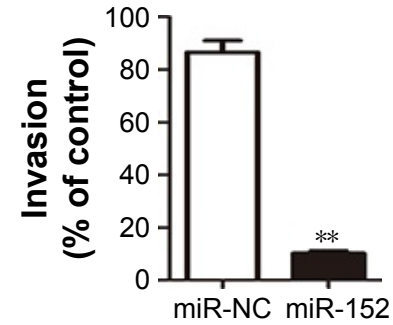

C
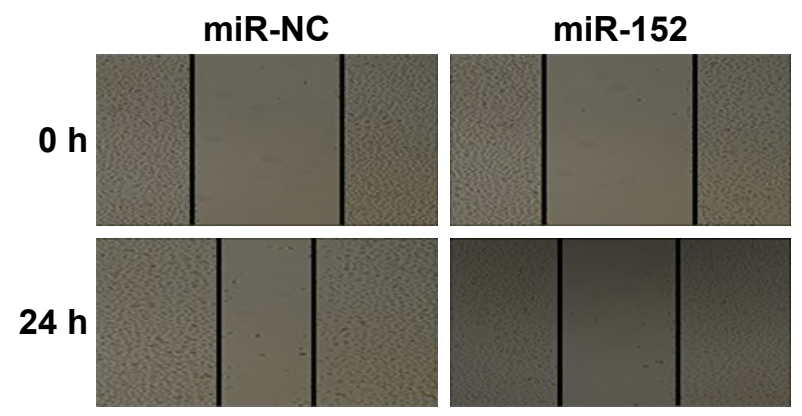

E

$\operatorname{miR}-152$
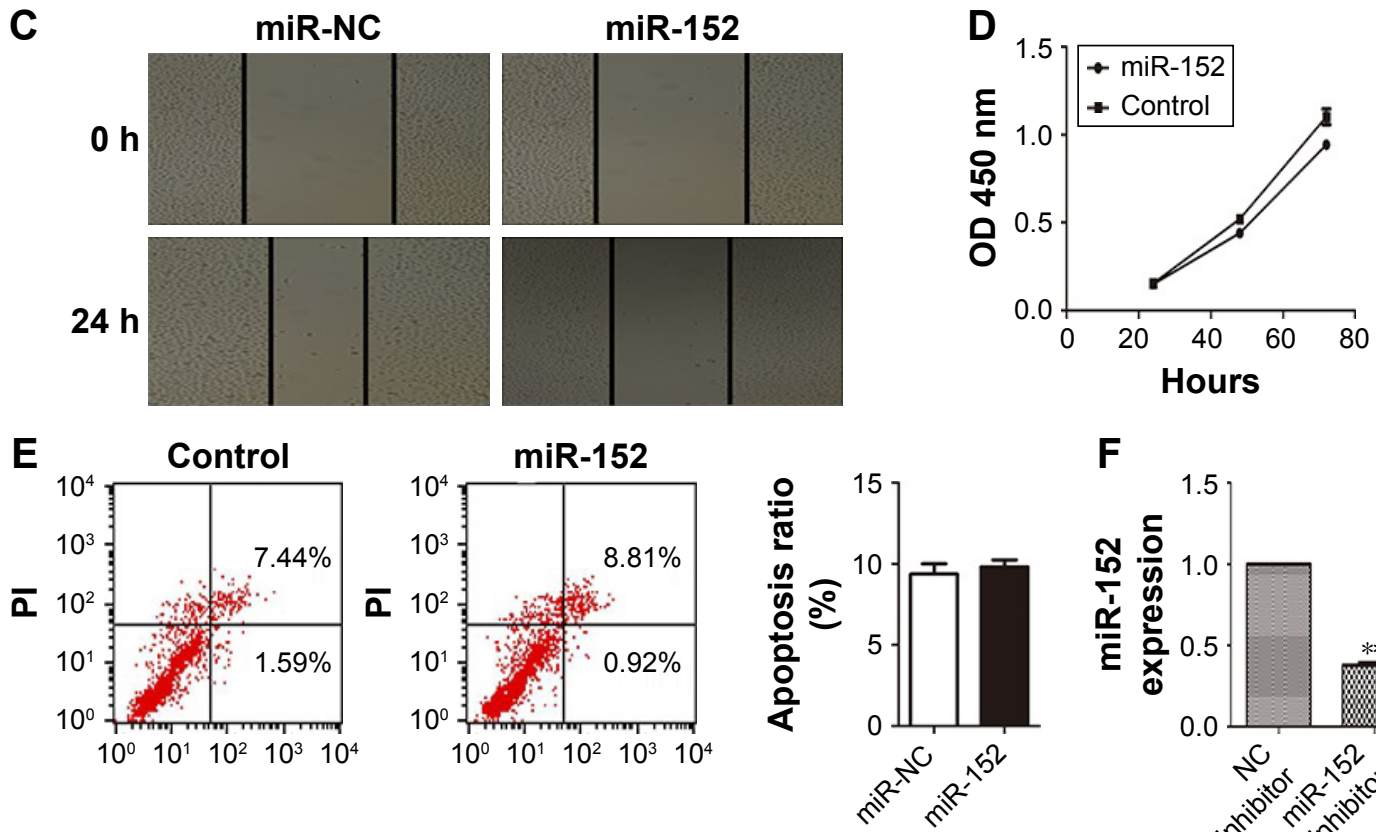

F
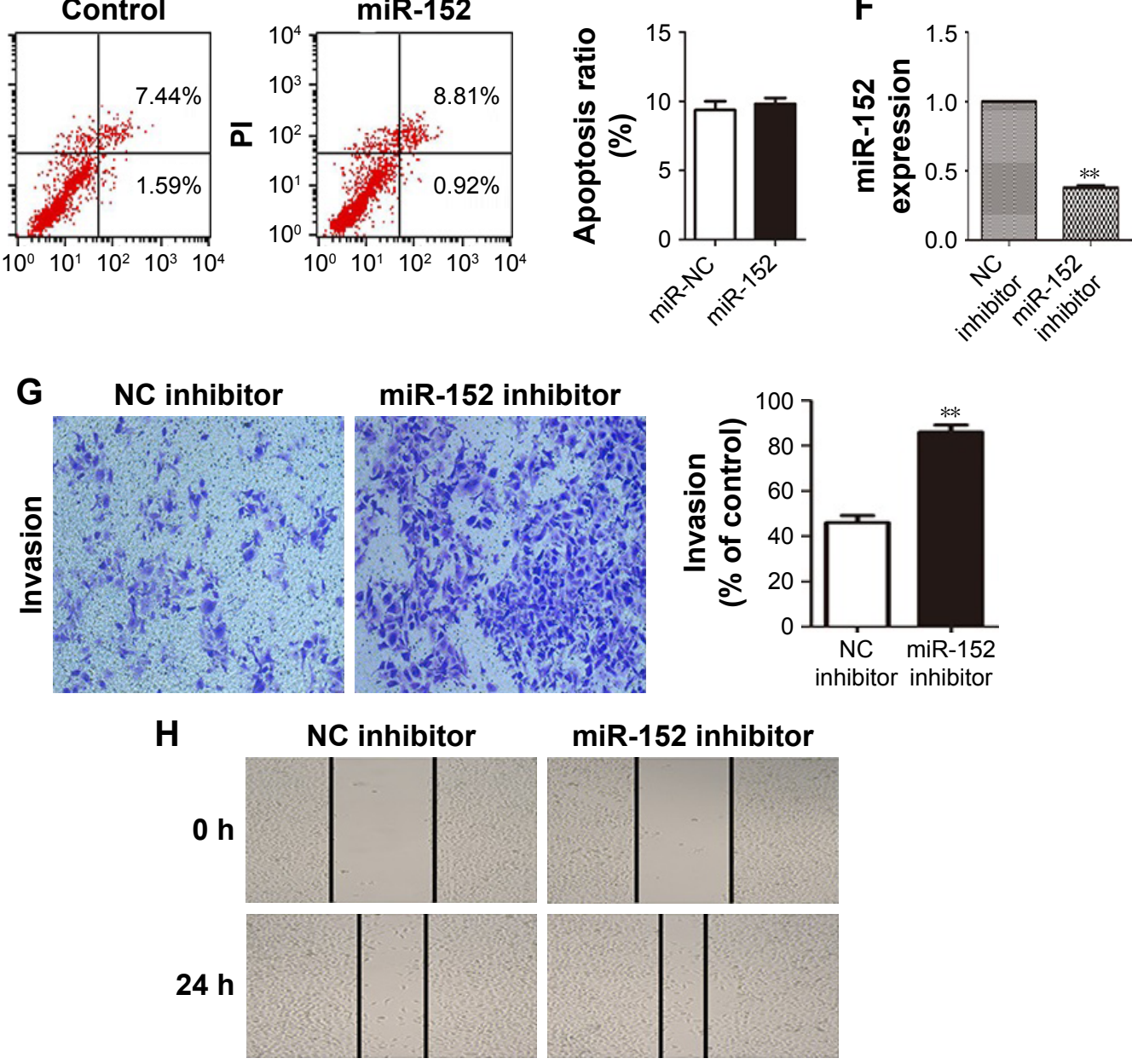

Figure 2 Overexpression of miR-152 inhibits the invasion and migration of NPC cells. (A) Expression of miR-I52 in CNE-2 cells transfected with negative control (miR-NC) or miR-I 52 mimic was detected using relative real-time PCR. *** $P<0.01$, compared with control. (B) Transwell assays demonstrated that restoration of miR-I52 suppressed invasion of CNE-2 cells. ${ }^{* * P}<0.01$, compared with control. (C) Wound healing assay showed that overexpression of miR-I52 significantly repressed the migration of CNE-2 cells. (D) Cell counting kit-8 assays showed that overexpression of miR-I52 had no effects on cell proliferation in CNE-2 cells. (E) Flow cytometry analysis showed that overexpression of miR-152 had no effects on cell apoptosis in CNE-2 cells. (F) Expression of miR-152 in CNE-2 cells transfected with negative control (NC inhibitor) or miR152 inhibitor was detected using relative real-time PCR. $* * P<0.0$ I, compared with control. (G) Transwell assays illustrated that downregulation of miR-I 52 promoted invasion of $\mathrm{CNE}-2$ cells. ${ }^{* * P}<0.01$, compared with control. $(\mathbf{H})$ Wound healing assay showed that downregulation of miR-I 52 significantly promoted the migration of $\mathrm{CNE}-2$ cells. Abbreviations: NPC, nasopharyngeal carcinoma; NC, negative control; PCR, polymerase chain reaction. 


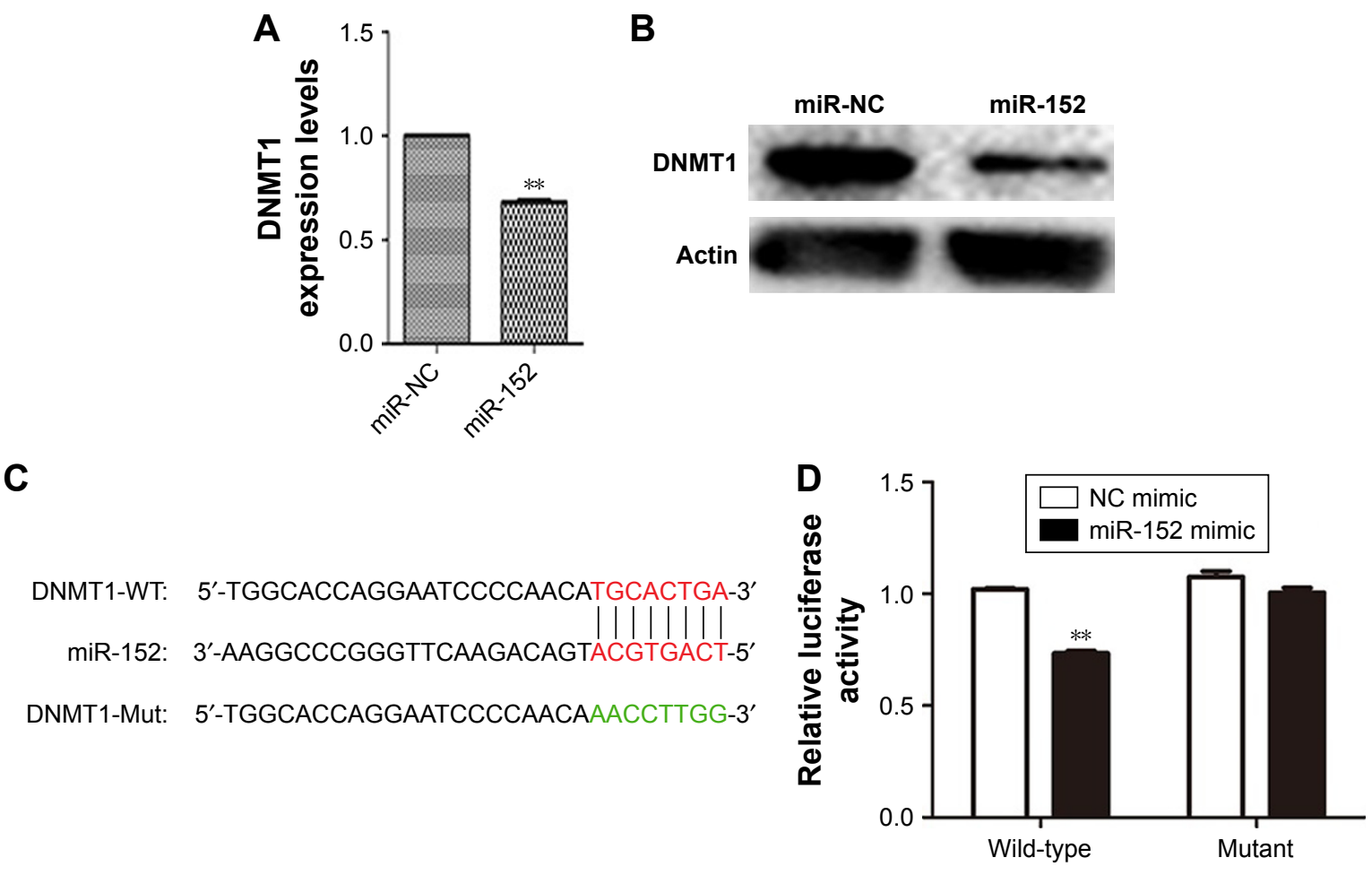

Figure 3 DNMTI is the direct target of miR-152. (A) Expression of DNMTI in the CNE-2 cells transfected with negative control (miR-NC) or miR-I52 mimic was detected using relative real-time PCR (B) Western blot. (C) Predicted human DNMTI 3'-UTR binding site for miR-I52. (D) MiR-I52 reduced luciferase activity of the wild-type, but had no effects on the mutant $3^{\prime}$-UTR of DNMTI. **P $<0.05$ compared with control.

Abbreviations: DNMTI, DNA (cytosine-5)-methyltransferase I; NC, negative control; PCR, polymerase chain reaction; WT, wild-type; Mut, mutant.

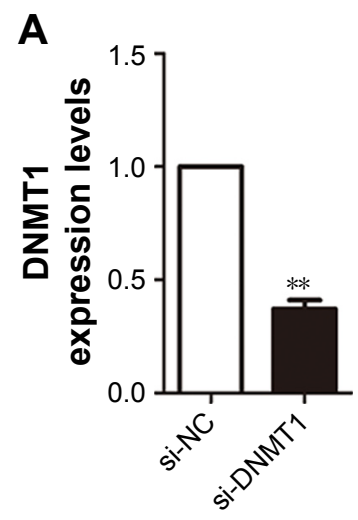

B

D

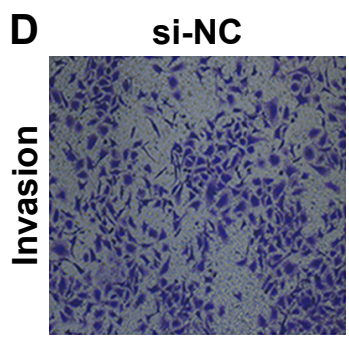

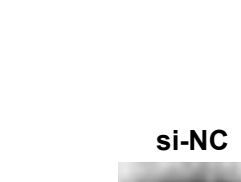

DNMT1

Actin

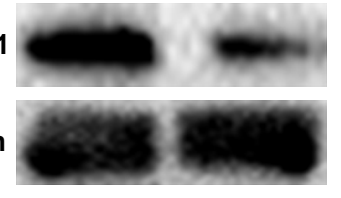

C

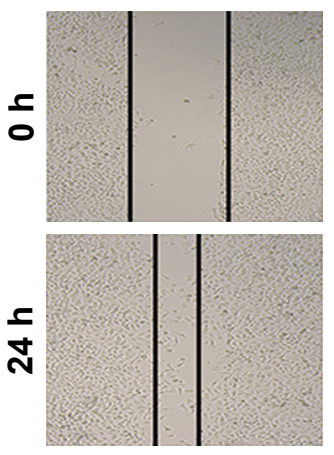

si-DNMT1

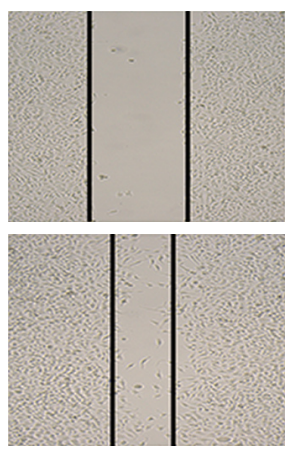

si-DNMT1
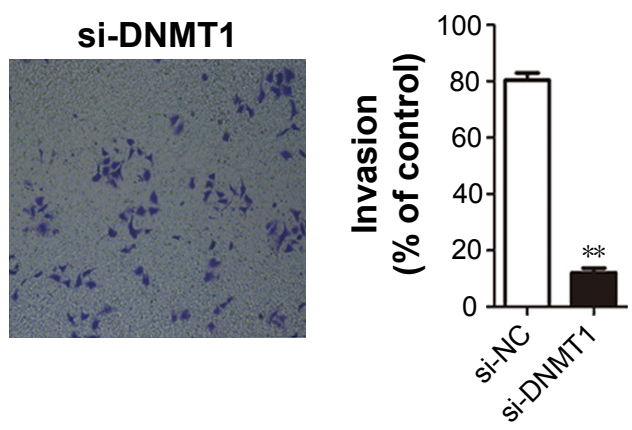

Figure 4 Knockdown of DNMTI significantly repressed the invasion and migration of NPC cells. (A) Efficiency of si-DNMTI was determined by qRT-PCR (B) Western blot in CNE-2 cells. (C) Wound-healing assays indicated that knockdown of DNMTI inhibited the migration capability of NPC cells. (D) Transwell assay showed that knockdown of DNMTI inhibited invasion capability of NPC cells. $* * P<0.05$ compared with control.

Abbreviations: DNMTI, DNA (cytosine-5)-methyltransferase I; NPC, nasopharyngeal carcinoma; PCR, polymerase chain reaction; NC, negative control. 


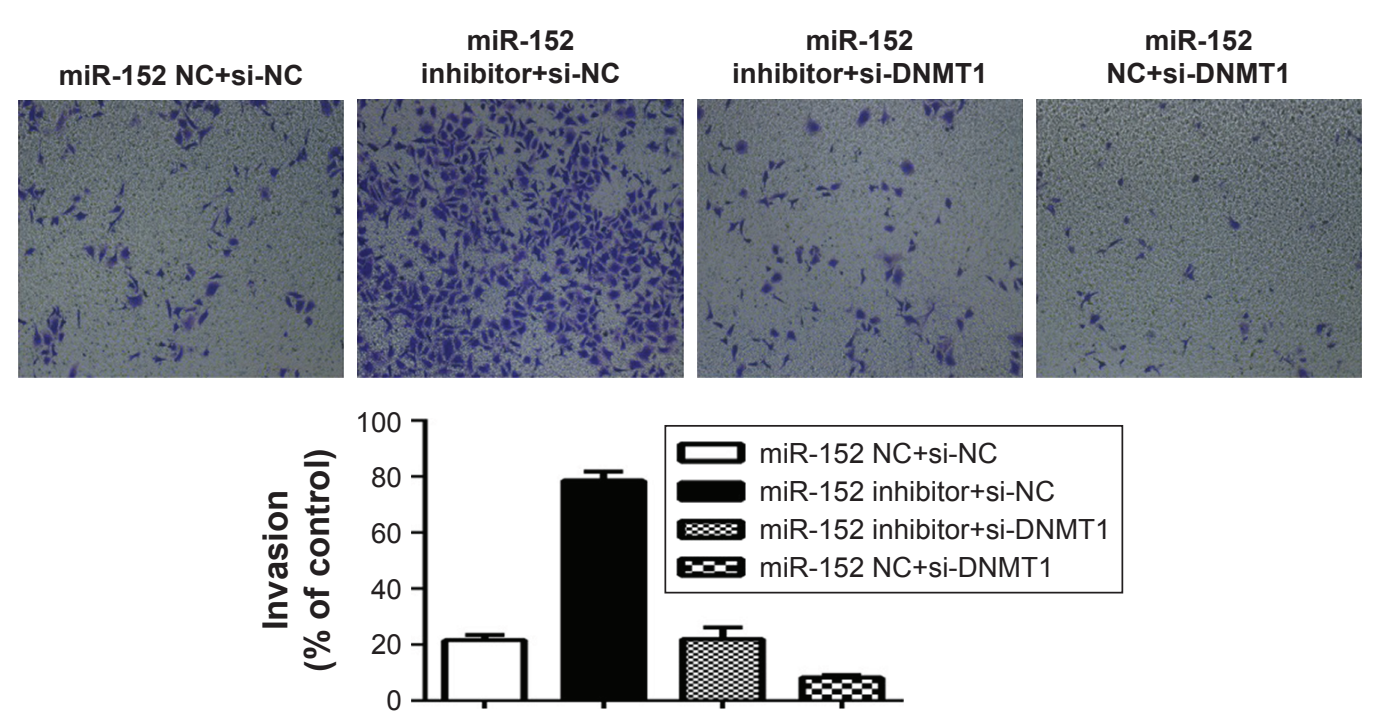

Figure 5 Knockdown of DNMTI reversed the effect of miR-152 inhibition on the invasion of NPC cells. Transwell analysis showed that the downregulation of DNMT I antagonized positive effects of miR-152 inhibitor on invasion of CNE-2 cells after the CNE-2 cells were transfected with miR-152 inhibitor or si-DNMTI.

Abbreviations: DNMTI, DNA (cytosine-5)-methyltransferase I; NPC, nasopharyngeal carcinoma; NC, negative control.

Inhibitor+si-NC, DNMT1-si+miR-152NC, and miR-152NC+ si-NC were designed for comparison. The results of matrigel invasion experiments showed that the inhibition of miR-152 expression increased cell migration ability, and the simultaneous silencing of DNMT1 can reverse this effect (Figure 5). Thus, miR-152 can act as a tumor suppressor in NPC cells by modulating DNMT1.

\section{DNMTI regulates miR-I52 expression through DNA methylation}

The regulatory circuit of miR-152 and DNMT1 was reported in several cancers, such as breast ${ }^{16}$ and prostate cancer. ${ }^{10}$ Silencing DNMT1 increased miR-152 expression in the CNE-2 cells (Figure 6A). Given that DNMT1 regulates methylation, we hypothesized that downregulating DNMT1 expression can attenuate the methylation level of miR-152 and consequently increases miR-152 expression. To verify this hypothesis, we transfected CNE-2 cells with DNMT1-SI. The methylation level of miR-152 was significantly decreased through pyrosequencing, and PCR results showed that miR-152 expression was elevated (Figure 6B). Furthermore, CNE-2 cells were treated with 5-aza-2'-deoxcycytidine. The DNA methylation level of miR-152 was gradually decreased with the increase of 5-aza- 2 -deoxcycytidine concentration $(0,10,20 \mu \mathrm{mol} / \mathrm{L}$; Figure $6 \mathrm{C})$, and its expression was upregulated as expected (Figure 6D). Consequently, our results show that the downregulation of DNMT1 expression inhibited the methylation level of miR-152 on its $\mathrm{CpG}$ islands, thereby increasing miR-152 expression.

\section{Discussion}

Owing to the developments in radiotherapy technology and treatment mode, local control and overall survival of NPC patients were effectively improved. ${ }^{17}$ However, the prognosis of $10 \%-20 \%$ patients with NPC remains poor because of local recurrence and distant metastasis. ${ }^{18,19}$ Exploring the molecular mechanisms of the invasion and metastasis in NPC may provide new effective strategies for the prevention, screening, and treatment of NPC.

Specific miRNAs are dysregulated in NPC, and play an important role in the progression and development of the disease. ${ }^{20}$ MiRNAs can be divided into two groups (according to their role in cancer), namely, oncogenes and tumor suppressor genes. ${ }^{5}$ Low miR-152 expression is frequently observed in most tumors; this condition suggests that miR-152 is a tumor suppressor in human cancer. ${ }^{6}$ However, miR-152 is upregulated in neuroblastoma and may act as an oncogene. ${ }^{21}$ In the present study, miR-152 expression was considerably reduced in NPC, as indicated by the GEO2R results. MiR-152 expression was downregulated in the six NPC cells. The upregulated miR-152 expression levels in the CNE-2 cells considerably inhibited cell invasion and migration, but had no significant effect on proliferation. We obtained opposite results when miR-152 expression was downregulated. All these results indicate that miR-152 plays a role as a tumor suppressor in NPC.

MiRNA exerts its function mainly by binding to the $3^{\prime} \mathrm{UTR}$ region of the target gene. ${ }^{22}$ In our study, we screened several target genes for miR-152 using TargetScan and finally 
A

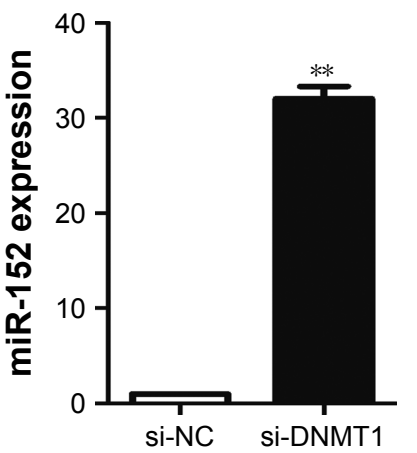

C
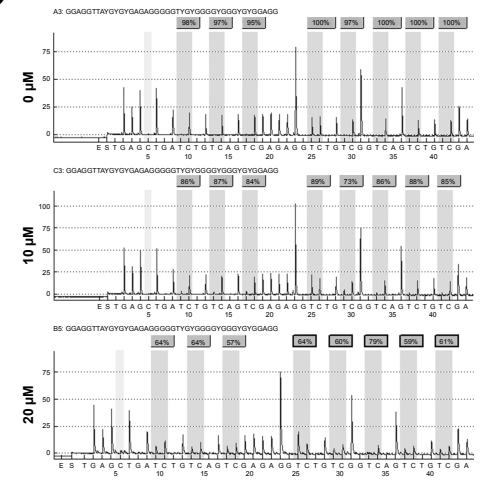

B
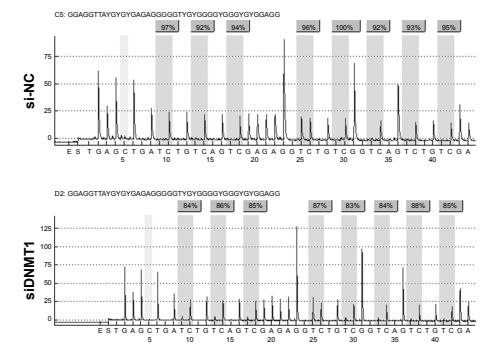

D

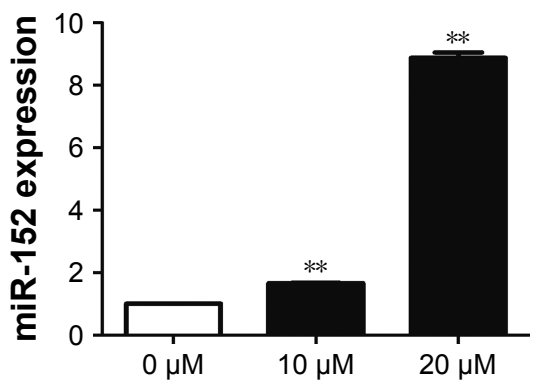

Figure 6 MiR-I52 can be regulated by DNMTI. (A) Downregulation of DNMTI increased the expression of miR-I52. (B) DNMTI attenuated methylation level of miR-152. (C) 5-aza-2'-deoxcycytidine can obviously reduce DNA methylation level of miR-152. (D) Expression of miR-152 was upregulated with the increase of 5-aza-2'deoxcycytidine concentration ( 0,10 , and $20 \mu \mathrm{mol} / \mathrm{L})$. $* * P<0.05$.

Abbreviations: DNMTI, DNA (cytosine-5)-methyltransferase I; NC, negative control.

selected DNMT1 according to the PCR results. DNMT1 is a methyltransferase and highly expressed in NPC. ${ }^{13}$ Previous studies showed that DNMT1 inhibits E-cadherin expression by increasing the rate of methylation of its promoter region, subsequently blocking NPC metastasis. ${ }^{23}$ In our study, increased miR-152 expression led to the downregulation of DNMT1 expression at mRNA and protein levels. We also confirmed that the knockdown of DNMT1 expression inhibited the invasion and migration of the NPC cells. This result was also observed when miR-152 expression was enhanced. Moreover, silencing of DNMT1 reverses the effect of miR-152 inhibition on the NPC cells. Luciferase assay results further confirmed that DNMT1 is the direct target of miR-152.

Abnormalities in genomic methylation may lead to the aberrant silencing of many tumor suppressor genes (TSGs), cellular functional genes, and miRNAs, and is, thus, closely related to the occurrence and development of cancer. ${ }^{14,24}$ Many publications revealed that miR-152, as a tumor suppressor, can be deactivated by methylation due to DNMT1. ${ }^{10,15}$ In the present study, the knockdown of DNMT1 increased miR-152 expression, indicating a negative feedback loop between miR-152 and DNMT1. Meanwhile, the level of miR-152 methylation decreased. Moreover, in the CNE-2 cells treated with 5-aza-2'-deoxcycytidine, the results were similar to those observed after knocking out DNMT1, but were more pronounced.

Increasing evidence showed that aberrant DNA methylation at promoter $\mathrm{CpG}$ islands of TSGs is common in NPC, particularly PCDH8, ${ }^{25}$ RASSF1A, ${ }^{26}$ and PCDH10 $2{ }^{27} \mathrm{MiR}-152$ may increase TSG expression by targeting DNMT1. MiR-152 might increase TSG expression by targeting DNMT1 so that it is involved in the tumorigenesis of NPC. E-cadherin was one of those TSGs which could also be modulated by DNMT1 because of methylation at its promoter region. ${ }^{13}$ To our knowledge, the loss of membranous E-cadherin expression can result in enhanced cell migration activity, because E-cadherin acts as an adhesion molecule that regulates cell-cell contact. ${ }^{23,28}$ Moreover, this phenomenon is also closely related to the invasion and metastasis of NPC. ${ }^{29}$ In our study, miR-152 could inhibit the migration and invasion of NPC by targeting DNMT1. From our observations, we proposed a hypothesis that downregulation of DNMT1 caused by miR-152 might lead to enhanced expression of E-cadherin, which is responsible for the inhibition of migration and invasion in NPC.

\section{Conclusion}

MiR-152 is downregulated in NPC and functions as a tumor suppressor by targeting DNMT1. In addition, a novel 
miR-152-DNMT1 regulatory circuit may provide a new epigenetic therapeutic target in NPC.

\section{Acknowledgments}

We thank the members of the Research Center of Clinical Oncology of Jiangsu province for their technical assistance. This work was supported by the Jiangsu Provincial Commission of Health and Family Planning Young Scholars Award (grant numbers Q201501); the Jiangsu Clinical Medicine Science and Technology Special Fund (grant number BL2014091); the National Natural Science Foundation of China (grant number 81672989); and the Medical Young Talent Foundation of Jiangsu Provincial Health Department (grant number QNRC2016648).

\section{Disclosure}

The authors report no conflicts of interest in this work.

\section{References}

1. Chou J, Lin YC, Kim J, et al. Nasopharyngeal carcinoma - review of the molecular mechanisms of tumorigenesis. Head Neck. 2008;30(7): 946-963.

2. Zhang L, Chen QY, Liu H, Tang LQ, Mai HQ. Emerging treatment options for nasopharyngeal carcinoma. Drug Des Devel Ther. 2013;7: 37-52.

3. Bensouda Y, Kaikani W, Ahbeddou N, et al. Treatment for metastatic nasopharyngeal carcinoma. Eur Ann Otorhinolaryngol Head Neck Dis. 2011;128(2):79-85.

4. Zamore PD, Haley B. Ribo-gnome: the big world of small RNAs. Science. 2005;309(5740):1519-1524.

5. Zhang B, Pan X, Cobb GP, Anderson TA. microRNAs as oncogenes and tumor suppressors. Dev Biol. 2007;302(1):1-12.

6. Liu X, Li J, Qin F, Dai S. miR-152 as a tumor suppressor microRNA: Target recognition and regulation in cancer. Oncol Lett. 2016;11(6): 3911-3916.

7. Zhai R, Kan X, Wang B, et al. miR-152 suppresses gastric cancer cell proliferation and motility by targeting CD151. Tumour Biol. 2014; 35(11):11367-11373.

8. Li B, Xie Z, Li B. miR-152 functions as a tumor suppressor in colorectal cancer by targeting PIK3R3. Tumour Biol. 2016;37(8):10075-10084.

9. Dang YW, Zeng J, He RQ, Rong MH, Luo DZ, Chen G. Effects of miR-152 on cell growth inhibition, motility suppression and apoptosis induction in hepatocellular carcinoma cells. Asian Pac J Cancer Prev. 2014; 15(12):4969-4976.

10. Theodore SC, Davis M, Zhao F, et al. MicroRNA profiling of novel African American and Caucasian Prostate Cancer cell lines reveals a reciprocal regulatory relationship of miR-152 and DNA methyltranferase 1. Oncotarget. 2014;5(11):3512-3525.

11. Li T, Chen JX, Fu XP, et al. microRNA expression profiling of nasopharyngeal carcinoma. Oncology Rep. 2011;25(5):1353-1363.
12. Hermann A, Goyal R, Jeltsch A. The Dnmt1 DNA-(cytosine-C5)methyltransferase methylates DNA processively with high preference for hemimethylated target sites. J Biol Chem. 2004;279(46): 48350-48359.

13. Tsai CL, Li HP, Lu YJ, et al. Activation of DNA methyltransferase 1 by EBV LMP1 involves c-Jun NH(2)-terminal kinase signaling. Cancer Res. 2006;66(24):11668-11676.

14. Jiang W, Cai R, Chen QQ. DNA methylation biomarkers for nasopharyngeal carcinoma: diagnostic and prognostic tools. Asian Pac J Cancer Prev. 2015;16(18):8059-8065.

15. Xu Q, Jiang Y, Yin Y, et al. A regulatory circuit of miR-148a/152 and DNMT1 in modulating cell transformation and tumor angiogenesis through IGF-IR and IRS1. J Mol Cell Biol. 2013;5(1):3-13.

16. Sengupta D, Deb M, Rath SK, et al. DNA methylation and not H3K4 trimethylation dictates the expression status of miR-152 gene which inhibits migration of breast cancer cells via DNMT1/CDH1 loop. Exp Cell Res. 2016;346(2):176-187.

17. Tang LL, Sun Y, Mao YP, et al. Prognostic value of parapharyngeal extension in nasopharyngeal carcinoma treated with intensity modulated radiotherapy. Radiother Oncol. 2014;110(3):404-408.

18. Chua MLK, Wee JTS, Hui EP, Chan ATC. Nasopharyngeal carcinoma. Lancet. 2016;387(10022):1012-1024.

19. Lee N, Harris J, Garden AS, et al. Intensity-modulated radiation therapy with or without chemotherapy for nasopharyngeal carcinoma: radiation therapy oncology group phase II trial 0225. J Clin Oncol. 2009;27(22):3684-3690.

20. Bruce JP, Liu FF. MicroRNAs in nasopharyngeal carcinoma. Chin J Cancer. 2014;33(11):539-544.

21. Liu DZ, Ander BP, Tian Y, et al. Integrated analysis of mRNA and microRNA expression in mature neurons, neural progenitor cells and neuroblastoma cells. Gene. 2012;495(2):120-127.

22. Huang T, Yin L, Wu J, et al. MicroRNA-19b-3p regulates nasopharyngeal carcinoma radiosensitivity by targeting TNFAIP3/NF-kappaB axis. J Exp Clin Cancer Res. 2016;35(1):188.

23. Zhang RL, Peng LX, Yang JP, et al. IL-8 suppresses E-cadherin expression in nasopharyngeal carcinoma cells by enhancing E-cadherin promoter DNA methylation. Int J Oncol. 2016;48(1):207-214.

24. Esteller M. Cancer epigenomics: DNA methylomes and histonemodification maps. Nat Rev Genet. 2007;8(4):286-298.

25. He D, Zeng Q, Ren G, et al. Protocadherin8 is a functional tumor suppressor frequently inactivated by promoter methylation in nasopharyngeal carcinoma. Eur J Cancer Prev. 2012;21(6):569-575.

26. Ye M, Huang T, Ni C, Yang P, Chen S. Diagnostic capacity of RASSF1A promoter methylation as a biomarker in tissue, brushing, and blood samples of nasopharyngeal carcinoma. EBioMedicine. 2017;18:32-40.

27. Ying J, Li H, Seng TJ, et al. Functional epigenetics identifies a protocadherin PCDH10 as a candidate tumor suppressor for nasopharyngeal, esophageal and multiple other carcinomas with frequent methylation. Oncogene. 2006;25(7):1070-1080.

28. Birchmeier W, Behrens J. Cadherin expression in carcinomas: role in the formation of cell junctions and the prevention of invasiveness. Biochim Biophys Acta. 1994;1198(1):11-26.

29. Ran Y, Wu S, You Y. Demethylation of E-cadherin gene in nasopharyngeal carcinoma could serve as a potential therapeutic strategy. J Biochem. 2011;149(1):49-54.
OncoTargets and Therapy

\section{Publish your work in this journal}

OncoTargets and Therapy is an international, peer-reviewed, open access journal focusing on the pathological basis of all cancers, potential targets for therapy and treatment protocols employed to improve the management of cancer patients. The journal also focuses on the impact of management programs and new therapeutic agents and protocols on

\section{Dovepress}

patient perspectives such as quality of life, adherence and satisfaction. The manuscript management system is completely online and includes a very quick and fair peer-review system, which is all easy to use. Visit http://www.dovepress.com/testimonials.php to read real quotes from published authors. 